\title{
In vitro Shoot Multiplication of Withania coagulans (Stocks) Dunal
}

\author{
Harsh Joshi, Sureshkumar Nekkala, Deepak Soner, Mafatlal M. \\ Kher and M. Nataraj*
}

P.G. Department of Biosciences, Sardar Patel University, Sardar Patel Maidan, Vadtal Road, Satellite Campus, Post Box No. 39, Vallabh Vidyanagar-388120, India

Key words: Shoot multiplication, Withania coagulans, Hyperhydricity

\begin{abstract}
Withania coagulans (Stocks) Dunal is an important medicinal plant of Solanaceae. Nodal segments obtained from field grown plants were used as explants. $1-2 \mathrm{~cm}$ long nodal segment with a single one bud was cultured on MS containing 2.5 $\mathrm{mg} / \mathrm{l}$ thidiazuron (TDZ), $0.1 \mathrm{mg} / \mathrm{l} \mathrm{NAA}$ and $50 \mathrm{mg} / \mathrm{l}$ adenine sulphate (AdS) resulted in formation of 5.16 shoots per node. However, vitrification was observed in all explants within one month. On the other hand nodal explants cultured on MS supplemented with $2.50 \mathrm{mg} / \mathrm{l}$ meta-topoline $(m \mathrm{~T})$ with $0.1 \mathrm{mg} / \mathrm{l}$ NAA and $50 \mathrm{mg} / \mathrm{l}$ AdS resulted in the formation of 4.50 healthy and uniformly grown shoots per node.
\end{abstract}

\section{Introduction}

Withania coagulans (Stocks) Dunal, commonly known as vegetable rennet, paneerbandh (Jain et al. 2012), or Indian cheese maker, belongs to Solanaceae. The fruits of $W$. cogulans are used as the source of coagulating enzyme for clotting the milk in the Punjab, which is called 'paneer' so it is also known as "Doda Paneer" (Gupta and Keshari 2013). Recent studies have shown anti-hyperglycemic activity of withanolide coagulin-L from W. coagulans (Beg et al. 2014). Its fruit extracts are reported to have antioxidant and anti-inflammatory activity (Ojha et al. 2014), while dried fruit extract has been reported to have anti-diabetic and anti-hyperlipidemic activity (Datta et al. 2013). Ethyl acetate extracts of $W$. coagulans have been reported to have leishmanicidal property (Kuroyanagi et al. 2012). Its other medicinal properties and ethanomedicinal values are well documented (Gupta 2012, Gupta and Keshari 2013, Jain et al. 2012). To avail

*Author for correspondence: <mnatarajspu@gmail.com>. 
elite genotypes, micropropagation is one of the methods for large scale propagation (Inamdar et al. 1990, Kher and Nataraj 2012, Kher et al. 2015, 2016, Nataraj et al. 2016, Varghese et al. 1993a,b, 1992). Micropropagation studies on W. cogulans is reviewed recently (Teixeira da Silva et al. 2015). Its natural propagation occurs through seeds, which is a slow process and resultant seeds may not germinate in the absence of favourable germination conditions (Rathore et al. 2012).

\section{Materials and Methods}

Young and actively growing branches of Withania coagulans $(15 \mathrm{~cm})$ were collected from Anand Agricultural University, Gujarat, India and rinsed in running tap water for $30 \mathrm{~min}$ to remove dirt. Thereafter the branches were soaked in 10\% liquid detergent for $10 \mathrm{~min}$ and thereafter were washed under running tap water for $10 \mathrm{~min}$ followed by thorough rinsing with tap water. Branches were cut into $5 \mathrm{~cm}$ long segments each with 3 - 4 nodes. Then these explants were treated with $0.1 \%(\mathrm{w} / \mathrm{v}) \mathrm{HgCl}_{2}$ for 3 min under aseptic conditions. These were then rinsed five times with sterile distilled water. These explants were then trimmed to an average size of $1.5-2.0 \mathrm{~cm}$ and inoculated in borosilicate test tube $(25 \times 150 \mathrm{~mm})$ containing $15 \mathrm{ml}$ of MS supplemented with $3 \%(\mathrm{w} / \mathrm{v}$ ) sucrose, $0.5,1.0,1.5,2.0$ and $2.5 \mathrm{mg} / \mathrm{l}$ of cytokinin, viz., BA, Kn, TDZ, $\mathrm{N}^{6}$-(2-isopentenyl) adenine (2-ip), zeatin or meta-topolin [6-(3hydroxybenzylamino) purine] $(\mathrm{mT})$ in combina-tion with $0.1 \mathrm{mg} / \mathrm{l} \mathrm{NAA}$ and 50 $\mathrm{mg} / \mathrm{l}$ adenine sulphate (AdS) for 21 days for culture initiation.

For subculturing, nodes of $1.5 \mathrm{~cm}$ from in vitro raised shoots derived from MS supplemented with $2.50 \mathrm{mg} / \mathrm{l} \mathrm{mT}, 0.1 \mathrm{mg} / \mathrm{l} \mathrm{NAA}$ and $50 \mathrm{mg} / \mathrm{l}$ AdS were isolated and inoculated in borosilicate glass bottle ( $400 \mathrm{ml}$ capacity) containing 50 $\mathrm{ml}$ of MS supplemented with BA [0.50 mg/l + 25, 50 or $75 \mathrm{mg} / \mathrm{l} \mathrm{AdS} \mathrm{and/or} \mathrm{yeast}$ extract (YE)]. In vitro raised shoots were on half strength MS, MS supplemented with $0.5,1.0,1.50$, and $2.00 \mathrm{mg} / \mathrm{l}$ NAA or IAA and $0.8 \%$ agar for rooting.

In vitro rooted plants were removed from culture vessel and gently washed under running tap water to remove adhering agar. Subsequently plantlets were transferred to plastic cups containing sterile sand, coco-peat and soil mixture in a $1: 1: 1$ ratio. The transplanted plants were covered with clear transparent plastic bags to maintain humidity, kept in the plant tissue culture laboratory, and watered with $15 \mathrm{ml}$ of one-fourth strength MS distilled water alternatively at 4 day intervals. After 10 days, the plastic bags were removed opened; and the uncovered plants were maintained at $25 \pm 2^{\circ} \mathrm{C}$ under a $16 / 8 \mathrm{hrs}$ light/dark photoperiod and $35 \mu \mathrm{mol} / \mathrm{m}^{2} / \mathrm{s}$ in the laboratory. After 30 days, plantlets were transferred to field under natural conditions. 
Throughout the study, MS was supplemented with various concentrations of $3 \%$ sucrose. The $\mathrm{pH}$ of the medium was adjusted to 5.8 and gelled with $0.8 \%$ agar. Cultures were kept at $16 / 8 \mathrm{hrs}$ photoperiod under cool white light provided by $36 \mathrm{~W}$ Philips tube at a light intensity of $35 \mu \mathrm{mol} / \mathrm{m}^{2} / \mathrm{s}$ and were maintained at $25 \pm 2^{\circ} \mathrm{C}$ room temperature.

All the experiments were conducted with a minimum 12 replicates per treatment and repeated twice. Each replicate represents one node per culture vessel. The results are expressed as means $\pm \mathrm{Sd}$ of two experiments. The data were analyzed statistically as one-way analysis of variance using SPSS version-19 (Chicago, IL. USA). The significance of difference among means values was carried out using DMRT (Duncan 1955) at $\mathrm{p}<0.05$.

\section{Results and Discussion}

The present study mainly focuses on high frequency axillary bud proliferation from $W$. coagulans for conservation (Figs 1A,F and 2A, B). Bud emergence from nodal explants started within 7 - 10 days in all plant growth regulators (PGRs), except 2-ip resulted in culture initiation within 10 - 15 days. It was observed that nodal explants cultured on MS supplemented with thidiazuron (1-phenyl-3-(1,2,3-thiadiazol-5-yl)urea) (TDZ), $50 \mathrm{mg} / \mathrm{l}$ AdS and 0.1 $\mathrm{mg} / \mathrm{l}$ NAA resulted in highest number of shoots as compared to other PGR treatments, but all resultant shoots were vitrified (Fig. 1C). However, nodal explants cultured on MS supplemented with $2.50 \mathrm{mg} / \mathrm{l}$ meta topoline, $50 \mathrm{mg} / \mathrm{l}$ AdS and $0.1 \mathrm{mg} / \mathrm{l} \mathrm{NAA}$, resulted in formation of healthy and shoots with uniform length (Fig. 1B) but numbers were comparatively less than TDZ supplemented medium (Table 1). Shoots obtained from $m \mathrm{~T}$ supplemented medium were significantly longer among all cytokinin treatments, including TDZ (Table 1). Shoots obtained from BA and Kn are smaller in number and their lengths were not uniform (Fig. 1A,E); they were stunted in growth in $0.50 \mathrm{mg} / 1$ TDZ supplemented medium (Fig. 1D). Vitrified shoots could not be used for rooting because it was very difficult to recover healthy plants from vitrified shoots, therefore TDZ was avoided in all subcultures and reduced concentrations of AdS and BA were used for subcultures of single nodes isolated from in vitro raised shoots of $W$. coagulans. The terminology "Hyperhydricity" formerly called "verification" has been used here to describe micropopagated plants with an abnormal morphological appearance and physiology (Debergh et al. 1992). Hyperhydricity, resulted from media composition and culture conditions like wounding, infiltration of soft culture medium, generally of a high ionic strength, 

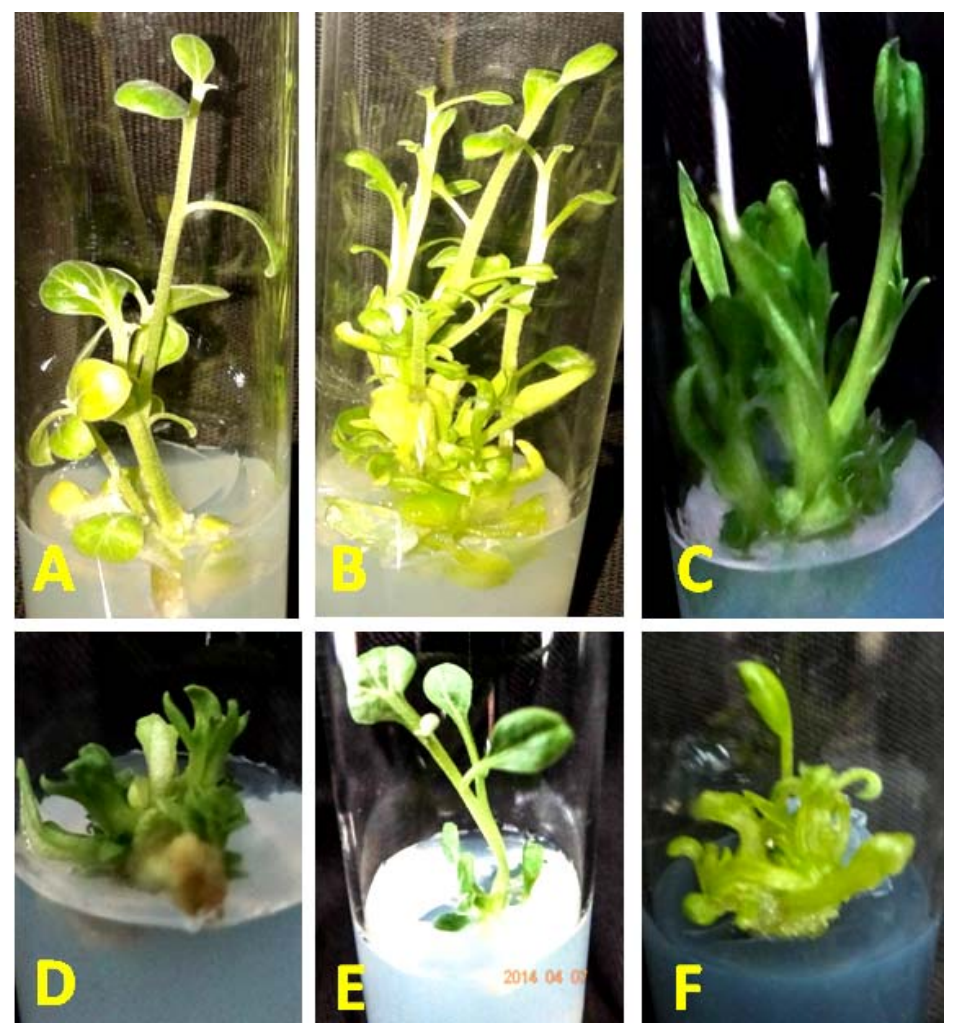

Fig. $1 \mathrm{~A}$ to E: Axillary shoot multiplication of $W$. coagulans where MS supplemented with $50 \mathrm{mg} / \mathrm{l}$ AdS and $0.1 \mathrm{mg} / 1$ NAA. A. Shoot multiplication of $W$. coagulans on MS supplemented with 2.50 $\mathrm{mg} / \mathrm{l}$ BA on 21st day with healthy shoots. B. Shoot multiplication from nodal explant of $W$. coagulans on MS supplemented with $2.50 \mathrm{mg} / 1 \mathrm{mT}$ on 21st day shows healthy and uniform shoots C. Shoot multiplication from nodal explant of $W$. coagulans on MS supplemented with $2.00 \mathrm{mg} / 1$ TDZ on 21st day, shoots were vitrified (abnormal shoots with elongated leaves). D. Shoot multiplication from nodal explant of $W$. coagulans on MS supplemented with TDZ $0.50 \mathrm{mg} / \mathrm{l}$ on 21st day having stunted growth and vitrified shoots. E. Shoot multiplication from nodal explant of $W$. coagulans on MS supplemented with $\mathrm{Kn} 2.50 \mathrm{mg} / \mathrm{l}$ on 21st day. F. Abnormal shoots (vitrified/ hyperhydric) were transferred on plant growth regulator free MS supplemented with $2.00 \mathrm{~g} / \mathrm{l}$ activated charcoal without plant growth regulators.

rich in nitrogen and in growth regulators in a special balance, in a humid and gaseous confined atmosphere (Kevers et al. 2004). High concentration of TDZ resulted in hyperhydricity of shoots reported in Pluchea lanceolata (Kher et al. 2014). TDZ at a higher concentration resulted in callus formation from leaf explants derived from in vitro raised shoots in $W$. coagulans is reported recently by Rathore et al. (2016). Effectiveness of $m \mathrm{~T}$ for axillary shoot multiplication and adventitious regeneration is reviewed by Aremu et al. (2011). 
Table 1. Effects of various concentrations of cytokinin in combination with $0.1 \mathrm{mg} / \mathrm{l} \mathrm{NAA}$ and $50 \mathrm{mg} / \mathrm{l}$ AdS on axillary shoot multiplications of W. coagulans.

\begin{tabular}{|c|c|c|c|c|c|c|c|c|c|}
\hline \multicolumn{6}{|c|}{ MS + PGRs combinations (mg/l) } & \multirow{2}{*}{$\begin{array}{l}\text { \% shoot } \\
\text { multipli- } \\
\text { cation }\end{array}$} & \multirow{2}{*}{$\begin{array}{l}\text { Number of } \\
\text { shoots/node } \\
\text { mean } \pm \text { Sd }\end{array}$} & \multirow{2}{*}{$\begin{array}{l}\text { Shoot length } \\
\text { in }(\mathrm{cm}) \\
\text { mean } \pm \text { Sd }\end{array}$} & \multirow{2}{*}{$\begin{array}{l}\text { Hyperhy- } \\
\text { dricity/ } \\
\text { vitrifica- } \\
\text { tion (\%) }\end{array}$} \\
\hline $\mathrm{BA}$ & $\mathrm{Kn}$ & TDZ & 2-ip & Zea & $m \mathrm{~T}$ & & & & \\
\hline- & - & - & - & - & - & 0 & $0 \mathrm{i}$ & $0 \mathrm{n}$ & \\
\hline 0.5 & - & - & - & - & - & 33.33 & $3.25 \pm 1.16$ cdefgh & $0.88 \pm 0.26 \mathrm{~m}$ & \\
\hline 1.0 & - & - & - & - & - & 41.67 & $2.80 \pm 1.14 \mathrm{gh}$ & $0.90 \pm 0.30 \mathrm{~lm}$ & \\
\hline 1.5 & - & - & - & - & - & 70.83 & $3.12 \pm 1.36$ defgh & $1.04 \pm 0.33 \mathrm{jklm}$ & \\
\hline 2.0 & - & - & - & - & - & 79.17 & $4.42 \pm 1.68 \mathrm{abc}$ & $1.27 \pm 0.40$ ghijkl & \\
\hline 2.5 & - & - & - & - & - & 79.17 & $4.68 \pm 1.25 \mathrm{ab}$ & $1.37 \pm 0.56$ ghij & \\
\hline- & 0.5 & - & - & - & - & 50.00 & $3.00 \pm 1.13$ efgh & $0.98 \pm 0.39 \mathrm{klm}$ & \\
\hline- & 1.0 & - & - & - & - & 54.17 & $2.69 \pm 1.18 \mathrm{gh}$ & $1.09 \pm 0.33 \mathrm{ijklm}$ & \\
\hline- & 1.5 & - & - & - & - & 58.33 & $3.21 \pm 1.72$ defgh & $1.34 \pm 0.51$ ghijk & \\
\hline- & 2.0 & - & - & - & - & 66.67 & $2.81 \pm 1.22 \mathrm{fgh}$ & $1.55 \pm 0.68 \mathrm{efg}$ & \\
\hline- & 2.5 & - & - & - & - & 58.33 & $3.86 \pm 1.46$ bcdefg & $1.97 \pm 0.55 \mathrm{~cd}$ & \\
\hline- & - & 0.5 & - & - & - & 66.67 & $4.69 \pm 0.95 \mathrm{ab}$ & $0.85 \pm 0.23 \mathrm{~m}$ & \\
\hline- & - & 1.0 & - & - & - & 70.83 & $4.29 \pm 1.49 \mathrm{abcd}$ & $1.11 \pm 0.30 \mathrm{ijklm}$ & $0-25$ \\
\hline- & - & 1.5 & - & - & - & 70.83 & $5.18 \pm 1.88 \mathrm{a}$ & $1.31 \pm 0.47$ ghijk & $25-50$ \\
\hline- & - & 2.0 & - & - & - & 75.00 & $4.94 \pm 1.76 \mathrm{ab}$ & $1.44 \pm 0.35 \mathrm{fghi}$ & $50-75$ \\
\hline- & - & 2.5 & - & - & - & 79.17 & $5.16 \pm 1.95 \mathrm{a}$ & $1.49 \pm 0.45 \mathrm{efgh}$ & $75-100$ \\
\hline- & - & - & 0.5 & - & - & 50.00 & $4.08 \pm 0.79$ abcdef & $0.98 \pm 0.39 \mathrm{klm}$ & \\
\hline- & - & - & 1.0 & - & - & 58.33 & $4.71 \pm 1.14 \mathrm{ab}$ & $1.04 \pm 0.33 \mathrm{jklm}$ & \\
\hline- & - & - & 1.5 & - & - & 66.67 & $4.69 \pm 1.08 \mathrm{ab}$ & $1.31 \pm 0.40$ ghijk & \\
\hline- & - & - & 2.0 & - & - & 75.00 & $4.67 \pm 1.03 \mathrm{ab}$ & $1.45 \pm 0.57 \mathrm{fghi}$ & \\
\hline- & - & - & 2.5 & - & - & 70.83 & $4.82 \pm 1.07 \mathrm{ab}$ & $1.63 \pm 0.64 \mathrm{defj}$ & \\
\hline- & - & - & - & 0.5 & - & 58.33 & $2.50 \pm 1.22 \mathrm{~h}$ & $0.97 \pm 0.45 \mathrm{klm}$ & \\
\hline- & - & - & - & 1.0 & - & 66.67 & $3.06 \pm 1.44 \mathrm{efgh}$ & $1.09 \pm 0.34 \mathrm{ijklm}$ & \\
\hline- & - & - & - & 1.5 & - & 75.00 & $3.22 \pm 1.31$ defgh & $1.14 \pm 0.31$ hijklm & \\
\hline- & - & - & - & 2.0 & - & 79.17 & $3.26 \pm 1.28$ cdefgh & $1.83 \pm 0.84$ cde & \\
\hline- & - & - & - & 2.5 & - & 83.33 & $3.30 \pm 1.26$ cdefgh & $1.75 \pm 0.55 \mathrm{def}$ & \\
\hline- & - & - & - & - & 0.5 & 5.17 & $3.77 \pm 1.09$ bcdefg & $1.64 \pm 0.74 \mathrm{defg}$ & \\
\hline- & - & - & - & - & 1.0 & 58.33 & $4.07 \pm 1.38$ abcde & $1.81 \pm 0.71 \mathrm{cde}^{\circ}$ & \\
\hline- & - & - & - & - & 1.5 & 66.67 & $4.00 \pm 1.55$ abcdef & $2.11 \pm 0.74 \mathrm{bc}$ & \\
\hline- & - & - & - & - & 2.0 & 70.83 & $4.29 \pm 1.36 \mathrm{abcd}$ & $2.40 \pm 0.90 \mathrm{ab}$ & \\
\hline- & - & - & - & - & 2.5 & 75.00 & $4.50 \pm 1.62 \mathrm{ab}$ & $2.70 \pm 1.17 \mathrm{a}$ & \\
\hline
\end{tabular}

Means \pm Sd value in each column followed by same letters are not significantly different according to DMRT at $\mathrm{p} \leq 0.05$. Data were recorded after 21 days.

During subculture, in vitro raised shoots from MS supplemented with 2.50 $\mathrm{mg} / \mathrm{l} \mathrm{mT}, 50 \mathrm{mg} / \mathrm{l}$ AdS and $0.1 \mathrm{mg} / \mathrm{l}$ NAA were used for experimental purpose, while data on other shoot cultures were not recorded. In addition to AdS, yeast extract (YE) alone or in combination with AdS was also tested for shoot multiplication of $W$. coagulans (Table 2). YE has been used as a supplement to promote plant growth due to the presence of amino acids (Thorpe et al. 2008). 
Table 2. Effects of adenine sulfate and yeast extract during subculture nodal explant derived from in vitro raised shoots of $W$. coagulans on MS supplemented with $0.50 \mathrm{mg} / 1 \mathrm{BA}$.

\begin{tabular}{lllll}
\hline $\begin{array}{l}\text { Adenine sulphate } \\
(\mathrm{mg} / \mathrm{l})\end{array}$ & $\begin{array}{l}\text { Yeast extract } \\
(\mathrm{mg} / \mathrm{l})\end{array}$ & Subculture & $\begin{array}{l}\text { No. of shoots/node } \\
\text { mean } \pm \mathrm{Sd}\end{array}$ & $\begin{array}{l}\text { Shoot length } \\
(\mathrm{cm}) \text { mean } \pm \mathrm{Sd}\end{array}$ \\
\hline 25 & 0.00 & 1 & $8.29 \pm 2.16 \mathrm{cde}$ & $2.98 \pm 0.56 \mathrm{~d}$ \\
50 & 0.00 & 1 & $9.58 \pm 3.28 \mathrm{c}$ & $3.71 \pm 0.70 \mathrm{c}$ \\
75 & 0.00 & 1 & $8.42 \pm 2.45 \mathrm{~cd}$ & $2.16 \pm 0.72 \mathrm{e}$ \\
0.00 & 25 & 1 & $6.88 \pm 2.13 \mathrm{e}$ & $2.33 \pm 0.31 \mathrm{e}$ \\
0.00 & 50 & 1 & $7.92 \pm 2.04 \mathrm{de}$ & $2.16 \pm 0.72 \mathrm{e}$ \\
0.00 & 75 & 1 & $8.29 \pm 2.16 \mathrm{cde}$ & $3.71 \pm 0.70 \mathrm{c}$ \\
25 & 0.00 & 2 & $17.04 \pm 2.96 \mathrm{~b}$ & $4.38 \pm 0.40 \mathrm{~b}$ \\
501 & 0.00 & 2 & $18.63 \pm 2.43 \mathrm{a}$ & $4.84 \pm 0.21 \mathrm{a}$ \\
75 & 0.00 & 2 & $17.17 \pm 2.22 \mathrm{ab}$ & $4.45 \pm 0.46 \mathrm{~b}$ \\
0.00 & 25 & 2 & $17.42 \pm 2.47 \mathrm{ab}$ & $3.71 \pm 0.70 \mathrm{c}$ \\
0.00 & 50 & 2 & $17.71 \pm 1.92 \mathrm{ab}$ & $4.51 \pm 0.41 \mathrm{~b}$ \\
0.00 & 75 & 2 & $17.54 \pm 1.91 \mathrm{ab}$ & $4.02 \pm 0.55 \mathrm{c}$ \\
\hline
\end{tabular}

Means \pm Sd value in each column followed by same letters are not significantly different according to DMRT at $\mathrm{p} \leq 0.05$. Data were recorded after 21 days.

Table 3. Effects of various concentrations auxins on rooting of W. coagulans.

\begin{tabular}{|c|c|c|c|c|c|c|}
\hline $\begin{array}{l}\text { IAA } \\
(\mathrm{mg} / \mathrm{l})\end{array}$ & $\begin{array}{l}\text { NAA } \\
(\mathrm{mg} / \mathrm{l})\end{array}$ & $\begin{array}{l}\text { MS } \\
\text { strength }\end{array}$ & $\begin{array}{l}\text { Percentage } \\
\text { response }\end{array}$ & $\begin{array}{l}\text { Number of roots } \\
\text { mean } \pm \text { Sd }\end{array}$ & $\begin{array}{l}\text { Root length } \\
(\mathrm{cm}) \text { mean } \pm \mathrm{Sd}\end{array}$ & $\begin{array}{l}\text { Callus } \\
\text { formation } \\
\% \\
\end{array}$ \\
\hline 0 & 0 & $1 / 2$ & 0.00 & $0 \mathrm{~d}$ & $0 \mathrm{f}$ & - \\
\hline 0.5 & 0 & $1 / 2$ & 33.33 & $1.25 \pm 0.5 \mathrm{c}$ & $1.15 \pm 0.21 \mathrm{cde}$ & - \\
\hline 1.0 & 0 & $1 / 2$ & 33.33 & $1.50 \pm 0.5 \mathrm{abc}$ & $1.37 \pm 0.17$ cde & - \\
\hline 1.5 & 0 & $1 / 2$ & 33.33 & $2.25 \pm 0.5 \mathrm{ab}$ & $1.82 \pm 0.46 \mathrm{bc}$ & - \\
\hline 2.0 & 0 & $1 / 2$ & 58.33 & $2.43 \pm 0.5 \mathrm{a}$ & $3.21 \pm 0.64 \mathrm{a}$ & - \\
\hline 0 & 0.5 & $1 / 2$ & 41.67 & $1.33 \pm 0.5 \mathrm{bc}$ & $0.90 \pm 0.10 \mathrm{e}$ & - \\
\hline 0 & 1.0 & $1 / 2$ & 33.33 & $1.50 \pm 0.5 \mathrm{abc}$ & $1.05 \pm 0.13 \mathrm{de}$ & - \\
\hline 0 & 1.5 & $1 / 2$ & 33.33 & $1.75 \pm 0.5 \mathrm{abc}$ & $1.23 \pm 0.17$ cde & - \\
\hline 0 & 2.0 & 1 & 25.00 & $1.33 \pm 0.5 b c$ & $1.77 \pm 0.31 \mathrm{bc}$ & - \\
\hline 0 & 0 & 1 & 0.00 & $0 \mathrm{~d}$ & $0 \mathrm{f}$ & $75-100$ \\
\hline 0.5 & 0 & 1 & 0.00 & $0 \mathrm{~d}$ & $0 \mathrm{f}$ & $75-100$ \\
\hline 1.0 & 0 & 1 & 0.00 & $0 \mathrm{~d}$ & $0 \mathrm{f}$ & $75-100$ \\
\hline 1.5 & 0 & 1 & 25.00 & $1.33 \pm 0.58 \mathrm{bc}$ & $1.53 \pm 0.25 \mathrm{~cd}$ & $25-50$ \\
\hline 2.0 & 0 & 1 & 33.33 & $2.0 \pm 0.82 \mathrm{abc}$ & $2.17 \pm 0.36 b$ & $25-50$ \\
\hline 0 & 0.5 & 1 & 0.00 & $0 \mathrm{~d}$ & $0 \mathrm{f}$ & $75-100$ \\
\hline 0 & 1.0 & 1 & 0.00 & $0 \mathrm{~d}$ & $0 \mathrm{f}$ & $75-100$ \\
\hline 0 & 1.5 & 1 & 0.00 & $0 \mathrm{~d}$ & $0 \mathrm{f}$ & $75-100$ \\
\hline 0 & 2.0 & 1 & 0.00 & $0 \mathrm{~d}$ & $0 \mathrm{f}$ & $75-100$ \\
\hline
\end{tabular}

Means \pm Sd value in each column followed by same letters are not significantly different according to DMRT at $\mathrm{p} \leq 0.05$. Data were recorded after 21st days. 


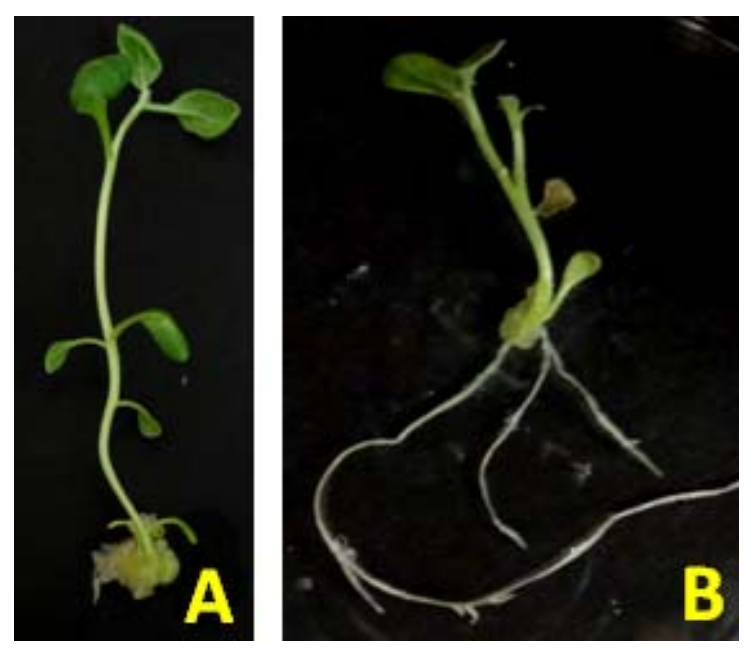

Fig. 2A. In vitro rooting in full strength MS supplemented with $2.0 \mathrm{mg} / 1 \mathrm{NAA}$ on 30 days of culture (shows callusing at the base of shoot), B. In vitro rooting in half strength MS supplemented with $2.0 \mathrm{mg} / \mathrm{l} \mathrm{NAA}$ on 30 days of culture.

In vitro raised shoots were successfully rooted on half strength MS supplemented with $2.0 \mathrm{mg} / \mathrm{l} \mathrm{NAA} \mathrm{(Fig.} \mathrm{2B),} \mathrm{while} \mathrm{callus} \mathrm{formation} \mathrm{was} \mathrm{observed}$ at the base of in vitro raised shoots (Fig. 2A) when inoculated on full strength MS medium supplemented with IAA or NAA (Table 3). In vitro raised plantlets were acclimated and transferred to field condition with $85 \%$ of survival rate. In vitro raised shoots can be useful for production of synthetic seeds for short term storage (Rathore and Kheni 2015).

\section{References}

Aremu AO, Bairu MW, Doležal K, Finnie JF and Van Staden J (2011) Topolins: A panacea to plant tissue culture challenges? Plant Cell, Tiss. Org. Cult. 108: 1-16. doi: 10.1007/s11240-011-0007-7

Beg M, Chauhan P, Varshney S, Shankar K, Rajan S, Saini D, Srivastava MN, Yadav PP and Gaikwad AN (2014) A withanolide coagulin-L inhibits adipogenesis modulating Wnt/ $\beta$-catenin pathway and cell cycle in mitotic clonal expansion. Phytomedicine 21: 406-414. doi: 10.1016/j.phymed.2013.10.009

Datta A, Bagchi C, Das S, Mitra, A., Pati, A. De, Tripathi SK and Datta, A (2013) Antidiabetic and antihyperlipidemic activity of hydroalcoholic extract of Withania coagulans Dunal dried fruit in experimental rat models. J. Ayurveda \& Integrative Medicine 4: 99. doi: 10.4103/0975-9476.113880

Debergh P, Aitken-Christie J, Cohen D, Grout B, von Arnold S, Zimmerman R and Ziv M (1992) Reconsideration of the term "vitrification" as used in micropropagation. Plant Cell, Tiss. Org. Cult. 30: 135-140. doi: 10.1007/BF00034307 
Duncan D (1955) Multiple range and multiple F tests. Biometrics 11: 1-42. doi: $10.2307 / 3001478$

Gupta PC (2012) Withania coagulans Dunal - An overview. International J. Pharmaceutical Sci. Review \& Research 12: 68-71.

Gupta V and Keshari BB (2013) Withania coagulans Dunal (Paneer Doda): A review. International J. Ayurvedic and Herbal Medicine 2: 1136-1144.

Inamdar JA, Nataraj M, Mohan JSS and Subramanian RB (1990) Somatic embryogenesis from callus cultures of Crataeva nurvala Buch. Ham. Phytomorphology 40: 319-322.

Jain R, Kachhwaha S and Kothari SL (2012) Phytochemistry, pharmacology, and biotechnology of Withania somnifera and Withania coagulans: A review. J. Medicinal Plants Research 6: 5388-5399. doi: 10.5897/JMPR12.704

Kevers C, Franck T, Strasser RJ, Dommes J and Gaspar T (2004) Hyperhydricity of micropropagated shoots: A typically stress-induced change of physiological state. Plant Cell, Tiss. Org. Cult. 77: 181-191. doi: 10.1023/B:TICU.0000016825.18930.e4

Kher MM, Joshi D, Nekkala S, Nataraj M and Raykundaliya DP (2014) Micropropagation of Pluchea lanceolata (Oliver \& Hiern.) using nodal explant. J. Hort. Research 22: 35-39. doi: 10.2478/johr-2014-0004

Kher MM and Nataraj M (2012) Micropropagation of Hibiscus radiatus - a medicinal plant. In: Braganza DV, Ghosh DSK, Menon DS (Eds) Impact of Plant Tissue Culture on Advances in Plant Biology. Loyola Center for Research and Development, Xavier's Research Foundation, Ahmedabad, India. pp. 18-26.

Kher MM, Nataraj M, Parmar HD and Buchad H (2015) Micropropagation of Merremia quinquefolia (L.) Hallier F. from nodal explants. J. Horticultural Research 23: 13-16. doi: 10.2478/johr-2015-0002

Kher MM, Nataraj M and Teixeira da Silva JA (2016) Micropropagation of Crataeva L. species. Rendiconti Lincei 27: 157-167. doi: 10.1007/s12210-015-0478-2

Kuroyanagi M, Murata M, Nakane T, Shirota O, Sekita S, Fuchino H and Shinwari ZK (2012) Leishmanicidal active withanolides from a Pakistani medicinal plant, Withania coagulans. Chemical \& Pharmaceutical Bulletin 60: 892-897. doi: 10.1248/cpb.c12-00264

Mishra S, Sangwan RS, Bansal S and Sangwan NS (2013) Efficient genetic transformation of Withania coagulans (Stocks) Dunal mediated by Agrobacterium tumefaciens from leaf explants of in vitro multiple shoot culture. Protoplasma 250: 451-458. doi: 10.1007/s00709-012-0428-0

Nataraj M, Kher MM and Teixeira da Silva JA (2016) Micropropagation of Clerodendrum species: A review. Rendiconti Lincei 27: 169-179. doi: 10.1007/s12210-015-0484-4

Ojha S, Alkaabi J, Amir N, Sheikh A, Agil A, Fahim MA and Adem A (2014) Withania coagulans fruit extract reduces oxidative stress and inflammation in kidneys of streptozotocin-induced diabetic rats. Oxidative Medicine and Cellular Longevity 2014: 1-9. doi: $10.1155 / 2014 / 201436$

Rathore MS and Kheni J (2015) Alginate encapsulation and in vitro plantlet regeneration in critically endangered medicinal plant, Withania coagulans (Stocks) Dunal. Proc. National Academy of Sciences, India Section B: Biological Sciences. doi: 10.1007/s40011-015-0577-y 
Rathore MS, Mastan SG, Yadav P, Bhatt VD, Shekhawat NS and Chikara J (2016) Shoot regeneration from leaf explants of Withania coagulans (Stocks) Dunal and genetic stability evaluation of regenerates with RAPD and ISSR markers. South African J. Botany 102: 12-17. doi: 10.1016/j.sajb.2015.08.003

Rathore MS, Shekhawat S, Kaur G, Singh RP and Shekhawat NS (2012) Micropropagation of vegetable rennet (Withania coagulans [Stocks] Dunal) - a critically endangered medicinal plant. J. Sustainable Forestry 31: 727-746. doi: 10.1080/ 10549811.2012.706533

Teixeira da Silva JA, Kher MM, Soner D and Nataraj M (2015) Withania coagulans (Stocks) Dunal: Biotechnological achievements and perspectives. J. Horticultural Research 23: 5-12. doi: 10.2478/johr-2015-0001

Thorpe TA, Stasolla C, Yeung EC, de Klerk GJ, Roberts AV and George, EF (2008) The components of plant tissue culture media ll. Organic additions, osmotic and $\mathrm{pH}$ effects, and support systems. In: George EF, Hall MA, de Klerk GJ (Eds) Plant propagation by tissue culture vol. 1. The background $3^{\text {rd }}$ Edn. Springer Netherlands, Dordrecht. pp. 115-173

Varghese SK, Inamdar JA, Kalia K, Subramanian RB and Nataraj M (1993a) In vitro organogenesis in Enicostema hyssopifolium (Willd) Verdoon. Proc. National Academy of Sciences, India Section B: Biological Sciences 63: 219-221.

Varghese SK, Inamdar JA, Kalia K, Subramanian RB and Nataraj M (1993b) Micropropagation of Aegle marmelos (L.) Corr. Phytomorphology 43: 87-92.

Varghese SK, Subramanian RB, Kalia K, Nataraj M and Inamdar JA (1992) In vitro studies on Vitex negundo. Indian Botanical Contactor 9: 147-150. 